\title{
Mecanismo sensor y de adaptación a los niveles de oxígeno y su implicancia en las enfermedades cardiovasculares: a propósito del Premio Nobel de Fisiología-Medicina 2019
}

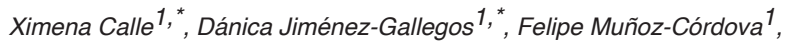 \\ Pablo Sánchez ${ }^{1}$, Sergio Lavandero ${ }^{1,2, \# .}$ \\ 1. Advanced Center for Chronic Diseases (ACCDiS), Facultad de Ciencias Químicas y Farmacéuticas y Facultad de Medicina, Universidad de \\ Chile, Santiago, Chile. \\ 2. Cardiology Division, Department of Internal Medicine, University of Texas Southwestern Medical Center, Dallas, Texas, USA.
}

${ }^{*}$ Ambas son primeras autoras y contribuyen de igual manera a este manuscrito.

El Premio Nobel 2019 en Fisiología-Medicina se confirió a los Profesores Gregg Semenza, William Kaelin y Sir Peter Ratcliffe por sus investigaciones en la maquinaria molecular que regula la expresión de genes sensibles a los cambios en los niveles de oxígeno. La síntesis de eritropoyetina inducida por la disminución de los niveles sanguíneos de oxígeno condujo al estudio del gen de la eritropoyetina y descubrimiento de los elementos de respuesta a hipoxia (HRE) en la región promotora y posteriormente al factor transcripcional inducible por hipoxia tipo 1 (HIF-1). Este factor consta de dos subunidades: HIF- $1 \alpha$, sensible al oxígeno, y HIF-1 $\beta$, expresada constitutivamente. HIF1 activa la transcripción de genes que codifican enzimas, transportadores y proteínas mitocondriales que disminuyen la utilización de oxígeno al cambiar el metabo- lismo oxidativo al metabolismo glicolítico y además aquellos involucrados en la angiogénesis y diferenciación celular. Las investigaciones paralelas en la enfermedad von Hippel-Lindau (VHL), un desorden autosómico dominante, permitieron descubrir el mecanismo de degradación de HIF1 en condiciones de normoxia y como se estabiliza bajo hipoxia. El impacto de HIF en clínica radica en el establecimiento de nuevas dianas terapéuticas para combatir la anemia y diversas enfermedades cardiovasculares. HIF promueve la angiogénesis a través de la expresión del factor de crecimiento vascular endotelial (VEGF), agente cardioprotector con potencial para tratar la isquemia/reperfusión, hipertrofia patológica e insuficiencia cardíaca.

Palabras clave: HIF-1, hipoxia, angiogénesis, ECV. 


\section{Role of hypoxia-inducible factor-1 (HIF-1) in the adaptacion to oxygen levels: Nobel Prize in physiology-medicine 2019}

The Nobel Prize in Physiology-Medicine was awarded to Drs. Gregg Semenza, William Kaelin and Sir Peter Ratcliffe for their research in the molecular machinery that regulates the expression of genes sensitive to the change in oxygen levels. The synthesis of erythropoietin induced by the decrease levels of oxygen in the blood led to investigate the promoter of the erythropoietin gene where the so-called hypoxia response elements (HRE) were described. Semenza et al. described a protein that binds to HREs and called it hypoxia-inducible transcriptional factor (HIF) that regulates gene expression among those involved in angiogenesis, cell differentiation and glycolytic en- zymes. HIF presents two oxygen-sensitive subunits HIF- $1 \alpha$ and HIF- $1 \beta$ constitutively expressed. In parallel, Kaelin et al. investigated von Hippel-Lindau disease (VHL), an autosomal dominant disorder, discovering a mutation of this protein generated a behavior similar to hypoxia. The impact of HIF-1 $\alpha$ lies in the search for new strategies such as hydrolase inhibitors to combat prevalent diseases, including anemia and cardiovascular diseases These compounds promote the expression of vascular endothelial growth factor (VEGF), a cardioprotective agent with potential use in pre- and post-conditioning therapy, cardiac hypertrophy and heart failure. 


\section{Premio Nobel en Fisiología-Medicina 2019}

El reciente Premio Nobel en esta área se confirió a los Dres. Gregg Semenza (Johns Hopkins University), William Kaelin Jr; (Harvard University) y Sir Peter Ratcliffe (Oxford University) por sus notables contribuciones para dilucidar cómo nuestras células sensan y se adaptan a los cambios en los niveles de oxígeno mediante la identificación de la maquinaria molecular esencial que regula la expresión de genes a dichos cambios. La importancia de sus descubrimientos radica en la promesa del desarrollo de nuevas estrategias para combatir la anemia, cáncer, enfermedades cardiovasculares y muchas otras patologías ${ }^{1,2}$.

\section{La necesidad del oxígeno en las células}

Desde hace varios siglos hemos reconocido que el oxígeno cumple un papel vital en el metabolismo energético mitocondrial en prácticamente todas las células de nuestro organismo, dado que se requiere en procesos bioquímicos claves que permiten la conversión oxidativa de los combustibles químicos presentes en nuestra dieta a energía (ATP). Otto Warburg, Premio Nobel de Fisiología-Medicina en 1931 describió los sistemas enzimáticos que forman el proceso respiratorio que media esta conversión. A lo largo de la evolución, un hito clave fue la aparición de oxígeno en nuestra atmósfera y su utilización por las primeras formas de vida. La teoría endosimbionte sostiene que esas primeras formas de vida colonizaron las células procariontes, permitiendo a las células vegetales y animales generar grandes cantidades de ATP mediante los cloroplastos y mitocondrias, respectivamente. Posteriormente, se desarrollaron en los seres pluricelulares mecanismos para asegurar un suministro basal de oxígeno a las células y tejidos. Los cuerpos carotideos, adyacentes a las grandes arterias en ambos lados del cuello, contienen células especializadas que sensan los niveles de oxígeno en la sangre. El Dr. Corneille Heymans (Premio Nobel de Fisiología y Medicina 1938) descubrió cómo los niveles sanguíneos de oxígenos son sensados por los cuerpos carotídeos, comunicándose directamente con el cerebro para controlar la frecuencia respiratoria. Sin embargo, a pesar de estos significativos avances, se desconocía hasta hace un par de décadas atrás los mecanismos moleculares de cómo las células sensan y se adaptan a cambios en los niveles de oxígeno ${ }^{1}$.

\section{Primeros descubrimientos: eritropoyetina e hipoxia}

Hoy sabemos que la eritropoyetina regula indirectamente los niveles de oxígeno al controlar la producción de nuevos glóbulos rojos a través de la eritropoyesis. Esta área de investigación se desarrolló a partir del hallazgo que una disminución del oxígeno en la sangre (hipoxia) inducia la síntesis de eritropoyetina por las células peritubulares del riñón ${ }^{3}$. Sin embargo, otro indicio clave para descubrir el mecanismo sensor de los niveles de oxígeno, surgió a partir de la evidencia que la intoxicación por cobalto aumentaba la masa de glóbulos rojos (eritrocitosis o policitemia). Inicialmente se pensó que la hipoxia sólo genera respuestas adaptativas en el hígado y riñón ${ }^{4,5}$, pero observaciones posteriores mostraron que otras células también se adaptaban dinámicamente a variaciones en los niveles de oxígeno. Así, Maxwell y Ratcliffe revelaron inicialmente que el promotor del gen de la eritropoyetina contenía secuencias nucleotídicas de 256 pares de bases específicas (hoy en día denominadas como hypoxia-responsive element, HRE) y que estas secuencias se encontraban también presentes en otros genes ${ }^{6}$. Las secuencias HRE son los sitios de reconocimiento y de unión para factores de transcripción activados bajo condiciones de anemia e hipoxia ${ }^{6}$. En 1992, Semenza y cols. descubrieron una proteína que se unía selectivamente a estas secuencias HRE y que denominaron factor transcripcional inducible por hipoxia (HIF). Este nuevo factor controla la expresión de diversos genes blancos, entre los se incluyen a aquellos que codifican a las enzimas glucolíticas 7 .

En forma paralela, Kaelin y cols. investigaron las causas de la enfermedad de von Hippel-Lindau (VHL), un raro desorden autosómico dominante con mutaciones en el gen VHL asociado a mayor riesgo de cáncer y un defecto en la proteína VHL origina un comportamiento similar a la hipoxia ${ }^{8}$. A partir de estos estudios, Ratcliffe y cols. describieron que VHL media la degradación de HIF-1 $\alpha$ y que una mutación en esta proteína mantiene HIF activo, mostrando que VHL interactúa físicamente con HIF-1 $\alpha$ y promueve su degradación en condiciones de normoxia 9 .

\section{Factor transcripcional HIF-1: estructura y regulación} HIF-1 es una familia de factores de transcripción que regulan la expresión de genes vinculados a la angiogénesis, diferenciación celular, metabolismo anaeróbico inducidos por la hipoxia ${ }^{10}$. HIF-1 es un heterodímero con dos subunidades HIF-1 $\alpha$ de $120 \mathrm{kDa}$ sensible al oxígeno y HIF $1 \beta$ también conocido como Translocador Nuclear de Receptor de Aril hidrocarburos (ARNT) de 91-94 kDa expresado constitutivamente e independiente de la disponibilidad del oxígeno y es estable en condiciones de normoxia ${ }^{12}$. Existen tres isoformas de HIF $\alpha$ conocidas: HIF- $1 \alpha$, HIF $2 \alpha$ y HIF-3 $\alpha$. La subunidades $\alpha$ son proteí- 


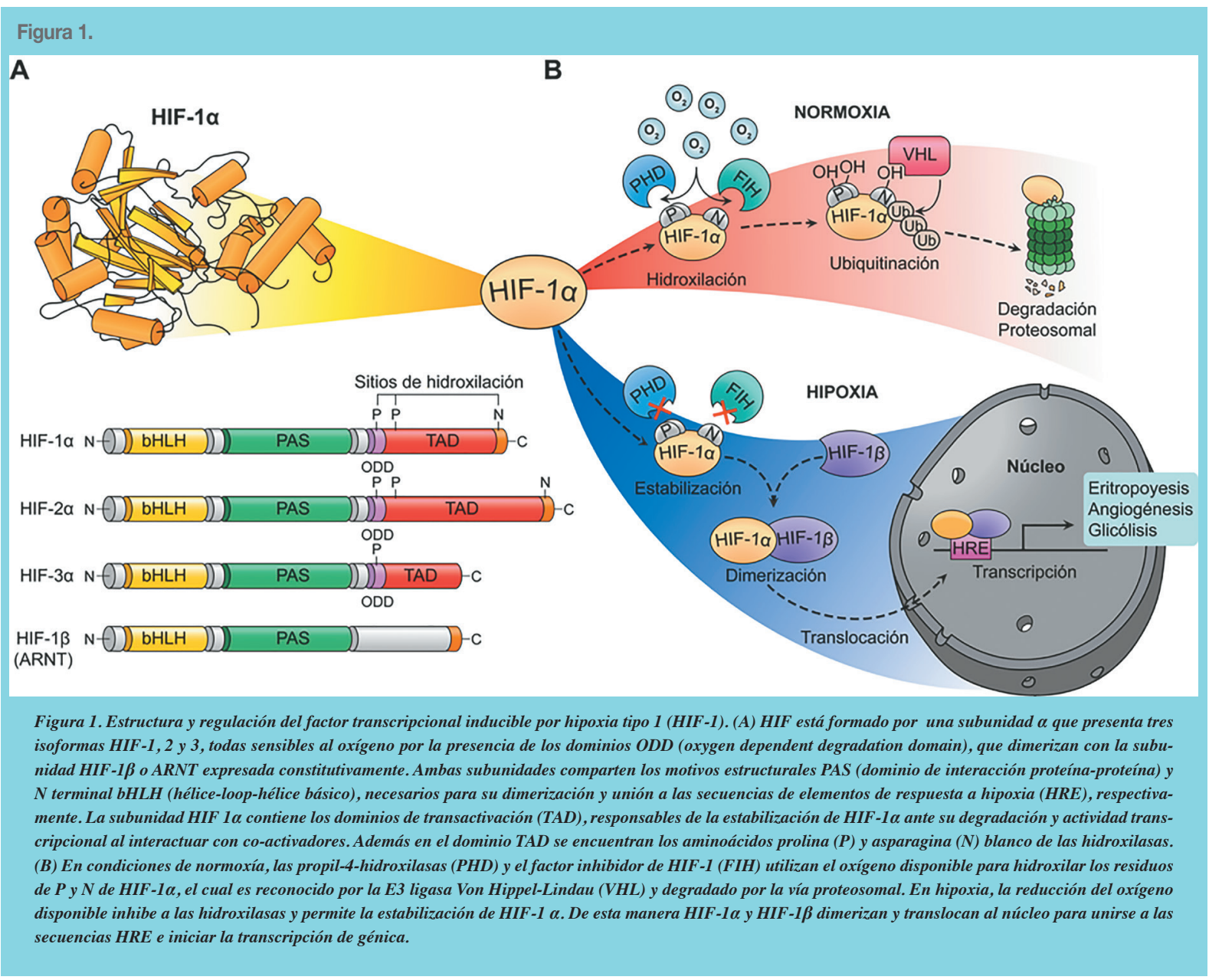

nas que tienen una estructura de helix loop-helix (bHLH), característica de varias familias de factores transcripcionales cuya actividad se regula por dimerización de sus subunidades, siendo en este caso esencial para su interacción con HIF-1 $\beta^{11}$. En cambio, HIF-1 $\alpha$ detecta el oxígeno y en su presencia conduce a su hidroxilación, poliubiquitinación y degradación por la vía proteosomal, resultando en una vida media de aproximadamente $5 \mathrm{mi}-$ nutos ${ }^{13}$. HIF- $\alpha$ contiene dos dominios de transactivación en los terminales amino y carboxilo así también un dominio de degradación dependiente de oxígeno (ODD) que contiene dos conservados residuos de prolina que regula su estabilidad dependiente del oxígeno. Una reducción en los niveles de oxígeno produce estabilización de HIF$1 \alpha$, siendo una de las respuestas a la hipoxia ${ }^{13,14}$.

Las otras isoformas tienen otras funciones específicas. HIF-2 $\alpha$ desempeña un papel clave en la hipoxia crónica $^{15}$ mientras que HIF-3 $\alpha$ actúa como regulador negativo de HIF-1 $\alpha$ y HIF-2 $\alpha^{16}$. HIF-2 $\alpha$, tiene una masa molecular de $118 \mathrm{kDa}$ y $48 \%$ de similitud con HIF-1 $\alpha$.
También presenta dos dominios de transactivación en N-terminal y C-terminal ${ }^{15}$. Sus genes blancos son diferentes y posee un mecanismo de regulación específico. HIF-3 $\alpha$ (73 kDa) posee sólo el dominio N-terminal y se expresa predominantemente en el timo, pulmón, cerebro, corazón y riñón adulto ${ }^{17}$. En normoxia, HIF-3 $\alpha$ se degrada por hidroxilación dependiente de oxígeno ${ }^{18}$, pero bajo condiciones de hipoxia actúa como un dominante negativo de HIF-1 $\alpha$ y HIF- $2 \alpha{ }^{19}$

La estabilidad de la proteína de HIF-1 $\alpha$ está regulada por la enzima propil 4 hidroxilasa (PHD) mediante la hidroxilación en residuos específicos de la prolina, seguido de ubiquitinación y degradación proteosomal ${ }^{20}$. Adicionalmente, HIF-1 $\alpha$ y HIF-2 $\alpha$ también son regulados por un segundo sistema enzimático sensible al oxígeno que depende de la hidroxilación de una asparagina por FIH (Factor Inhibiting HIF-1) en el dominio de activación de HIF- $\alpha$ y que conduce a la inactivación de su actividad transcripcional. Esta reacción de hidroxilación es inhibida por cobalto y permite explicar las primeras observa- 


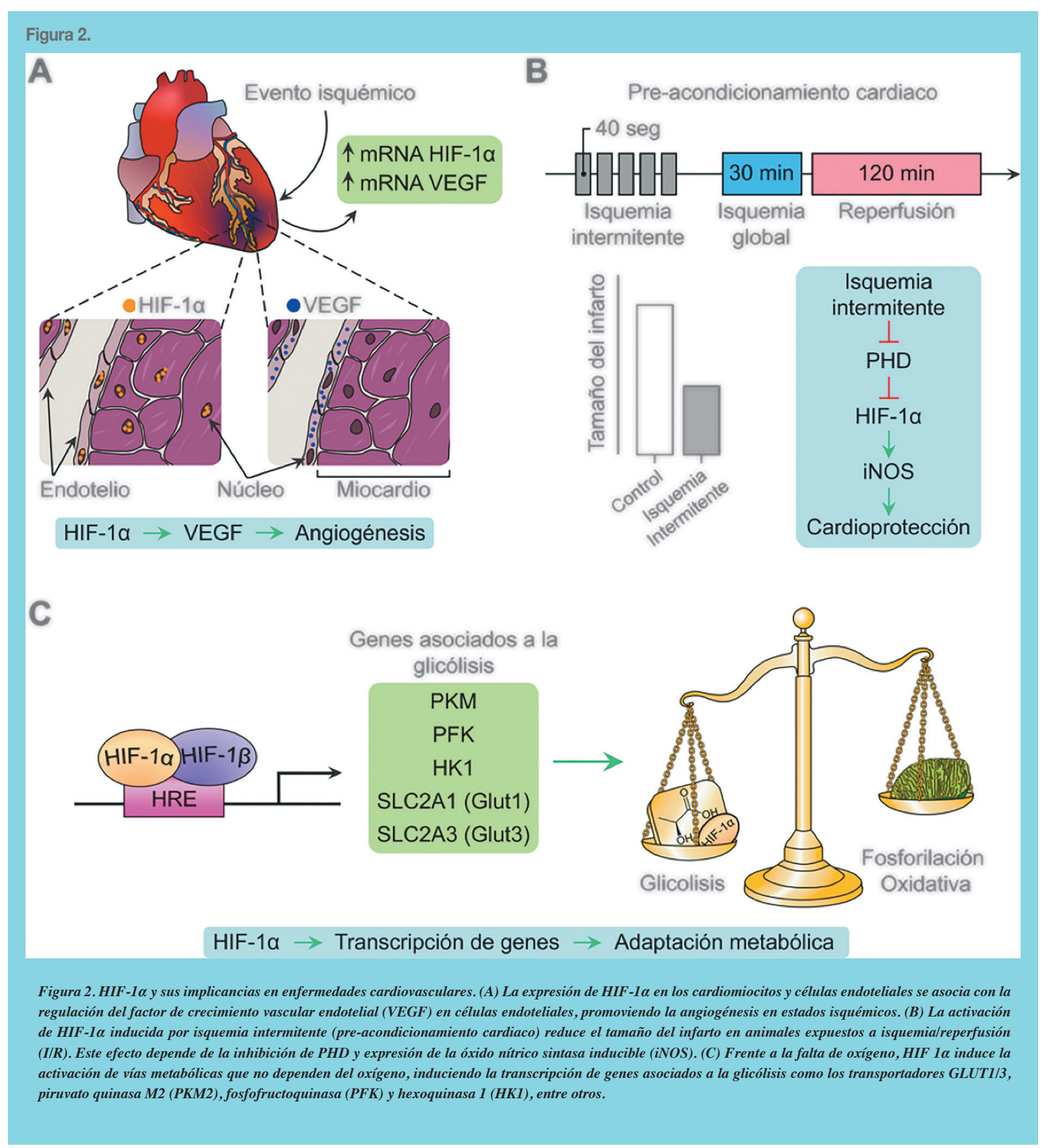

ciones en torno a los efectos de la intoxicación con este compuesto $^{11}$.

Tanto PHD como FIH introducen un grupo hidroxilo en HIF- $\alpha$, específicamente en dos residuos de prolina en el dominio ODD, requiriendo $\alpha$-cetoglutarato, hierro y oxígeno molecular y así estimula la interacción de HIF- $\alpha$ con la proteína (VHL). En condiciones de normoxia, la hidroxilación de HIF- $\alpha$ por PHD permite el reclutamiento de VHL E3 ligasa, poliubiquitinación y degradación de HIF-1 $\alpha^{21}$. Sin embargo, bajo hipoxia, las enzimas PHD inhiben la hidroxilación de HIF- $\alpha$, y, a su vez, impiden la unión de $\mathrm{pVHL}$, conduciendo a la estabilización de HIF- $\alpha$ y su translocación al núcleo donde se heterodimeriza con HIF $1 \beta$. Este complejo activa a los genes que poseen secuencias HRE en sus promotores $20,22,23$.

\section{HIF-1 y enfermedades cardiovasculares}

Las enfermedades cardiovasculares son la principal cau- 
sa de muerte a nivel mundial y en Chile ${ }^{24}$. Siendo una de las principales funciones del sistema cardiovascular el suministro de oxígeno, por tanto, ateroesclerosis una enfermedad altamente prevalente vinculada a una oxigenación insuficiente en los tejidos ${ }^{25}$ puede aumentar el riesgo de eventos cardiovasculares como infarto de miocardio o accidentes cerebro vasculares. Estudios mostraron que HIF-1 $\alpha$ participa en la respuesta compensatoria frente a estos daños, funcionando como un regulador transcripcional de diversos genes que median la respuesta vascular a la hipoxia y a la isquemia.

En efecto, HIF-1 promueve la expresión de los factores angiogénicos: factor de crecimiento vascular endotelial (VEGF), factor de crecimiento derivado de placenta (PGF), factor de crecimiento derivado de plaquetas tipo beta (PDGF- $\beta$ ), angiopoyetinas 1 y 2 (Angtp1 y Angpt2) El análisis de estos genes en cardiomiocitos, fibroblastos, células endoteliales vasculares y células del musculo liso vascular mostraron que cada tipo celular tiene un patrón diferente de expresión génica en respuesta a la hipoxia. HIF-1 además de promover la disponibilidad de oxígeno también activa la transcripción de genes que codifican enzimas, transportadores y proteínas mitocondriales que disminuyen la utilización de oxígeno al cambiar el metabolismo oxidativo al metabolismo glicolítico ${ }^{26,27}$. Ante una perfusión tisular reducida como respuesta fisiológica se induce la actividad de HIF-1, que activa la transcripción de genes que codifica factores angiogénicos como VEGF. Este factor estimula el reclutamiento de células endoteliales hacia el sitio de hipoxia e induce su proliferación (angiogénesis y vasculogénesis), también induce expresión de genes codificantes para metaloproteinasas (MMP) que controlan el metabolismo de la matriz extracelular y maduración de los vasos sanguíneos en condiciones de hipoxia 28,29 .

El trabajo de Lee Sang et al. ${ }^{30}$ enroló 37 pacientes infartados (27 hombres y 10 mujeres, edad promedio 65,9 años), a los cuales se les tomó una biopsia ventricular de las zonas infartadas y no infartadas como control. Los pacientes se estratificaron en los siguientes grupos: a) infarto temprano, b) infarto en evolución y c) estado de isquemia agudo. Un análisis molecular de las biopsias de la zona infartada mostró un aumento de HIF-1 $\alpha$ en los tres grupos de pacientes, mientras que solo en los grupos de evolución de infarto e isquemia aguda hubo un aumento en la expresión de VEGF, en comparación a las muestras control en todos los casos. Esto da a entender que HIF-1 $\alpha$ estaría regulando de forma temprana la expresión génica de las respuestas compensatorias ante estados hipóxicos y su expresión se mantendría en el tiempo para poder promover la expresión de VEGF y que este pueda preservar el tejido cardíaco en estados avanzados isquémicos. Además, identificaron la localización de ambas proteínas mediante inmunohistoquímicas, revelando que HIF-1 $\alpha$ se encontraba en el núcleo de los cardiomiocitos y células endoteliales que rodeaban las terminaciones de vasos sanguíneos pequeños y VEGF que sólo se apreció en el citoplasma de las células endoteliales que rodeaban estas terminaciones de los vasos sanguíneos. Desde aquí se empezó a proyectar que HIF $1 \alpha$ podría estar regulando otros genes sensibles a hipoxia además de VEGF. Por otro lado en un estudio en un modelo de ratón de isquemia de extremidades de 8 y 13 meses evaluaron el efecto del envejecimiento y HIF-1 concluyendo que afecta a la remodelación vascular inducida por la isquemia al inhibir la inducción de HIF-1 y su genes, bloqueando la señales angiogénicas ${ }^{31}$.

\section{HIF-1 media cardioprotección inducida por el pre- condicionamiento}

El precondicionamiento isquémico es una estrategia cardioprotectora contra la isquemia/reperfusión (I/R), que consiste en la activación de mecanismos endógenos de sobrevida provocados por breves episodios de isquemia transitoria y reperfusión ${ }^{32}$. Este concepto ha evolucionado hacia el condicionamiento isquémico que incluye varias estrategias cardioprotectoras endógenas, entre las que propone a HIF-1 como cardioprotector en terapias de pre condicionamiento y post-condicionamiento frente a I/R. Algunos estudios clínicos han mostrado efectos beneficiosos del pre condicionamiento al disminuir el daño por reperfusión en pacientes infartados ${ }^{33,34}$. La cardioprotección mediada por HIF-1 se podría explicar a través de la regulación de la expresión del gen de la óxido nítrico sintasa inducible (iNOS). Esta enzima está encargada de la producción de óxido nítrico (NO), siendo esencial para para el efecto cardioprotector del precondicionamiento en la fase tardía ${ }^{35}$. Otro estudio mostró la perdida completa del efecto cardioprotector del precondicionamiento en $\mathrm{I} / \mathrm{R}$ prolongada en ratones con deficiencia parcial de HIF-1 $\alpha^{36}$. Originalmente se postuló que la respuesta del pre condicionamiento implicaba principalmente al cardiomiocito. Sin embargo, investigaciones posteriores revelaron que la desactivación de HIF-1 $\alpha$ y HIF-1 $\beta$ en las células endoteliales del corazón también afecta a la cardioprotección $^{37}$. Se conoce que HIF-1 $\alpha$ promueve la transcripción de genes relacionados con vías metabólicas menos dependientes de oxígeno, lo cual es imprescin- 
dible para mantener funciones celulares en la isquemia. HIF $1 \alpha$ induce la transcripción de genes asociados a la glicolisis (transportadores GLUT 1-3, gliceraldehído 3 fosfato deshidrogenasa (GAPDH), aldolasa tipo C (AldoC) y la piruvato quinasa M2 (PKM2) ${ }^{38}$. También HIF$1 \alpha$ regula la oxidación de ácidos grasos, promoviendo la acumulación de lípidos por sobre su catabolismo, ya que la $\beta$-oxidación de ácidos grasos es más demandante de oxígeno que la oxidación de otros sustratos como la glucosa ${ }^{39}$. En la misma línea, existe evidencia que la activación de HIF-1 $\alpha$ fomenta el catabolismo de la fructosa en un proceso mediado por la inducción del factor de splicing 3B1 (SF3B1), el cual generar la isoforma C de la ceto hexoquinasa (KHK-C), como producto de la modificación postraduccional del transcrito del gen KHK. Este evento explica el aumento de la acidificación del medio extracelular (ECAR), lo cual es indicativo del metabolismo glicolítico anaerobio y disminución del consumo de oxígeno y menor fosforilación oxidativa bajo condiciones de hipoxia ${ }^{40}$.

El trabajo de Gui et al, ${ }^{41}$ informó, por primera vez, que HIF $1 \alpha$ regula la autofagia del cardiomiocito, promoviendo degradación de proteínas mediante la vía lisosomal para estimular la viabilidad celular frente a la hipoxia. La hipoxia también puede oxidar proteínas, causar estrés del retículo endoplásmico y activar la "respuesta a proteínas mal plegadas" (Unfolded Protein Response, UPR). Esta respuesta se activa en corazones de ratones infartados y en cultivo de cardiomiocitos bajo condiciones de hipoxia ${ }^{42,43}$. Interesantemente, algunos trabajos han vinculado el estrés de retículo por hipoxia con una mayor actividad de HIF-1 ${ }^{44}$. Sin embargo, existen estudios contradictorios ${ }^{45}$ por lo que se requieren nuevas investigaciones que clarifiquen dicha asociación.

\section{Implicancias de HIF-1 $\alpha$ en la hipertrofia e insuficien- cia cardíaca}

Existe evidencia que relaciona HIF- $1 \alpha$ al desarrollo de la hipertrofia cardíaca. Sus niveles proteicos están aumentados en muestras de corazón de pacientes con remodelado hipertrófico y con insuficiencia cardiaca terminal. A través de la generación de un modelo murino transgénico de HIF-1 $\alpha$ constitutivamente estabilizado, se describió que su activación temprana es benéfica para mantener la función cardíaca en el contexto de la hipertrofia. Sin embargo, su activación crónica es deletérea, contribuyendo al remodelado metabólico patológico del corazón hacia la glicolisis ${ }^{46}$. El aumento en la estabilización de HIF$1 \alpha$, mencionado anteriormente, también se ha observado en modelos experimentales de hipertrofia cardíaca patológica $^{39}$. También HIF1 parece mediar el desarrollo de esteatosis cardíaca asociada a cardiomiopatía hipertrófica por un mecanismo dependiente de PPAR $\gamma$, regulador maestro de la captación y anabolismo de lípidos a nivel cardiaco $^{39}$. Otras evidencias sugieren que HIF-1 $\alpha$ media el efecto de estímulos pro-hipertróficos como es el caso del péptido HIMF (factor mitogenico asociado a la hipoxia $)^{47}$. Otros datos vinculan a HIF-1 $\alpha$ con la insuficiencia cardiaca inducida por sobrecarga crónica de presión, efecto que es revertido por carvedilol. Se ha especulado que HIF-1 $\alpha$ podría mediar los efectos benéficos de este fármaco en la insuficiencia cardíaca. Sin embargo, aún esta posibilidad es prematura y se requiere validar esta premisa ${ }^{48}$. Otras evidencias en modelos experimentales animales han descrito cambios en los niveles de HIF $1 \alpha$ durante la transición de hipertrofia cardiaca patológica a insuficiencia cardiaca. El trabajo pionero de Sano y cols ${ }^{49}$ mostró que los niveles proteicos de HIF$1 \alpha$ aumentan durante la primera fase del desarrollo de la hipertrofia cardiaca por sobrecarga de presión (hasta las dos semanas), probablemente para favorecer la angiogénesis, compensar la hipoxia presente en el corazón hipertrófico y mantener la función contráctil. Sin embargo, una sobrecarga de presión sostenida (mayor a dos semanas) aumenta la concentración de las especies reactivas del oxígeno y daño al DNA, generándose acumulación y activación del factor transcripcional p53 que interacciona con HIF-1, promueve su degradación y cese del proceso de angiogénesis. Sin embargo, los niveles elevados de p53 también estimulan la muerte del cardiomiocito por activación del programa de apoptosis, caracterizado por aumento de los niveles de la proteína pro-apoptótica Bax. La mayor muerte de cardiomiocitos dependiente de la sobrecarga crónica de presión da cuenta de la progresión de la insuficiencia cardiaca 49 .

\section{Implicancias clínicas de HIF-1 $\alpha$}

El descubrimiento de HIF-1 $\alpha$ como el regulador maestro de la adaptación celular a la falta de oxígeno lo postula como un perfecto candidato para el tratamiento de la hipoxia aguda (intervenciones quirúrgicas o eventos isquémicos) y crónica (estenosis o insuficiencia vascular y secuelas post-operatorias $)^{50}$. La utilización de moduladores de la función de HIF-1 $\alpha$ ha tenido escasos resultados en el ámbito clínico, principalmente por el reducido tamaño de pacientes estudiados ${ }^{51}$, presencia de comorbilidades que interfieren con la terapia o por interacción con anestésicos u otros fármacos ${ }^{52}$. 
La sobre-expresión de HIF-1 $\alpha$ en tejidos humanos ha mostrado ser ineficiente o poco concluyente, sin cambios significativos respecto al grupo control tanto en músculo esquelético $^{53}$ como en corazón ${ }^{51}$, lo que contrasta radicalmente con los cambios positivos observados en animales ${ }^{54}$. Estos resultados podrían deberse al efecto compensatorio de las hidroxilasas de HIF-1 que favorecían su rápida degradación. Este antecedente ha motivado el desarrollo de inhibidores de las hidroxilasas de HIF1 (PDH), a fin de "quitar su freno" y favorecer su acción ${ }^{50}$, presentado ventajas potenciales como costo, vía de administración (oral), perfil de niveles sanguíneos de hierro mejorado y la producción endógena de EPO a un nivel cercano al rango fisiológico. Los tratamientos con EPO recombinante tiene una elevada incidencia de ECV, por lo que estos inhibidores de PHD podrían reducir este riesgo al estimular la eritropoyesis en ausencia de altas concentraciones de EPO. Por otra parte, algunos inhibidores de PHD reducen los niveles de colesterol y presión arterial pero aún no se ha identificado sus mecanismos ${ }^{25}$. El uso de estos fármacos ha tenido una respuesta positiva en ensayos clínicos para el tratamiento de la anemia ${ }^{55-57}$. Sin embargo, ninguno se realizado en condiciones de isquemia cardiaca aguda debido a la limitada ventana temporal para que el fármaco realice su efecto.

En la actualidad se ha desarrollado un método menos invasivo, conocido como pre condicionamiento isquémico remoto, consistente en ciclos de isquemia intermitente en lechos vasculares lejanos (por ejemplo, en una extremidad) con efectos cardioprotectores. Hasta la fecha se han aprobado numerosos ensayos clínicos en fase II con esta metodología, asociados a intervenciones pre-operatorias que muestran una reducción de los marcadores de daño cardiaco posterior a la operación ${ }^{58,59}$. Sin embargo, se requieren estudios de fase III dada la contradictoria evidencia $^{60}$. En conclusión, el efecto cardioprotector de la modulación de HIF-1 $\alpha$ en ensayos clínicos es poco concluyente y son necesarios más estudios para demostrar mejoras significativas en la sobrevida y calidad de vida de los pacientes.

\section{Agradecimientos:}

Este trabajo fue apoyado por el proyecto FONDAP 15130011 (S.L.)

\section{Referencias}

1. The Nobel Prize in Physiology or Medicine 2019.. https:// www.nobelprize.org/prizes/medicine/2019/press-release/

2. GARVEY C, TIEP B, CARTER R, BARNETT M, HART M, CASABURI R. Severe exercise-induced hypoxemia. Respir Care. 2012;57:1154-60. http://www.ncbi.nlm.nih.gov/pubmed/10315398.

3. KRANTZ SB. Erythropoietin. Blood. 199;77:419-34. http:// www.ncbi.nlm.nih.gov/pubmed/1991159.

4. PUGH CW, RATCLIFFE PJ. New horizons in hypoxia signaling pathways. Exp Cell Res. 2017; 356:116-21. https://www.
sciencedirect.com/science/article/pii/S0014482717301143?via\%3Dihub.

5. CASADEVALL N, MAYEUX P, LACOMBE C. L'érythropoḯtine. Physiologie, dosage, pathologie. Immuno-analyse Biol Spécialisée. 1989;4:7-IN2. https://www.sciencedirect. com/science/article/pii/S0923253289800845.

6. MAXWELL PH, PUGH CW, RATCLIFFE PJ. Inducible operation of the erythropoietin $3 \square$ enhancer in multiple cell lines: Evidence for a widespread oxygen-sensing mechanism. Proc Natl Acad Sci USA. 1993;90:2423-7. 
7. SEMENZA GL, ROTH PH, FANG HM, WANG GL. Transcriptional regulation of genes encoding glycolytic enzymes by hypoxia-inducible factor 1. J Biol Chem. 1994;269:23757-63. http://www.ncbi.nlm.nih.gov/pubmed/8089148.

8. LEDFORD H, CALLAWAY E. Biologists who decoded how cells sense oxygen win medicine Nobel. Nature. 2019;574:1612. http://www.nature.com/articles/d41586-019-02963-0

9. MAXWELL PH, WIESENER MS, CHANG G-W, CLIFFORD SC, VAUX EC, COCKMAN ME, et al. The tumour suppressor protein VHL targets hypoxia-inducible factors for oxygen-dependent proteolysis. Nature 1999;399:271-5. http://www.ncbi. nlm.nih.gov/pubmed/10353251.

10. SEMENZA GL. Hypoxia-inducible factors: mediators of cancer progression and targets for cancer therapy. Trends Pharmacol Sci. 2012;33:207-14. http://www.ncbi.nlm.nih.gov/pub$\operatorname{med} / 22398146$.

11. WANG GL, JIANG BH, RUE EA, SEMENZA GL. Hypoxia-inducible factor 1 is a basic-helix-loop-helix-PAS heterodimer regulated by cellular O2 tension. Proc Natl Acad Sci U S A. $1995 ; 92: 5510-4$.

12. WANG F, SHI S, ZHANG R, HANKINSON O. Identifying target genes of the aryl hydrocarbon receptor nuclear translocator (Arnt) using DNA microarray analysis. Biol Chem. 2006;387:1215-8. http://www.ncbi.nlm.nih.gov/pubmed/16972789.

13. SALCEDA S, CARO J. Hypoxia-inducible factor $1 \alpha$ (HIF- $1 \alpha)$ protein is rapidly degraded by the ubiquitin-proteasome system under normoxic conditions. J Biol Chem. 1997;272:22642-7. http://www.ncbi.nlm.nih.gov/pubmed/9278421.

14. HUANG LE, GU J, SCHAU M, BUNN HF. Regulation of hypoxia-inducible factor 1 is mediated by an O2-dependent degradation domain via the ubiquitin-proteasome pathway. Proc Natl Acad Sci USA. 1998;95:7987-92. http://www.ncbi. nlm.nih.gov/pubmed/9653127.

15. KOH MY, LEMOS R, LIU X, POWIS G. The hypoxia-associated factor switches cells from HIF-1 - to HIF-2 -dependent signaling promoting stem cell characteristics, aggressive tumor growth and invasion. Cancer Res. 2011;71:4015-27. http:// www.ncbi.nlm.nih.gov/pubmed/21512133.

16. YAMASHITA T, OHNEDA O, NAGANO M, IEMITSU M, MAKINO Y, TANAKA $\mathrm{H}$, et al. Abnormal heart development and lung remodeling in mice lacking the hypoxia-inducible factor-related basic helix-loop-helix PAS protein NEPAS. Mol Cell Biol. 2008;28:1285-97. http://www.ncbi.nlm.nih.gov/ pubmed/18070924.
17. GU YZ, MORAN SM, HOGENESCH JB, WARTMAN L, BRADFIELD CA. Molecular characterization and chromosomal localization of a third alpha-class hypoxia inducible factor subunit, HIF3alpha. Gene Expr 1998;7:205-13. http://www. ncbi.nlm.nih.gov/pubmed/9840812.

18. ZHANG P, YAO Q, LU L, LI Y, CHEN P-J, DUAN C. Hypoxia-inducible factor 3 is an oxygen-dependent transcription activator and regulates a distinct transcriptional response to hypoxia. Cell Rep 2014;6:1110-21. http://www.ncbi.nlm. nih.gov/pubmed/24613356.

19. MAKINO Y, CAO R, SVENSSON K, BERTILSSON G, ASMAN M, TANAKA H, et al. Inhibitory PAS domain protein is a negative regulator of hypoxia-inducible gene expression. Nature. 2001;414:550-4. http://www.ncbi.nlm.nih.gov/pubmed/11734856

20. JAAKKOLA P, MOLE DR, TIAN Y-M, WILSON MI, GIELBERT J, GASKELL SJ, et al. Targeting of HIF-alpha to the von Hippel-Lindau ubiquitylation complex by o2-regulated prolyl hydroxylation. Science. 2001;292:468-72. http://www.ncbi. nlm.nih.gov/pubmed/11292861.

21. SEMENZA GL. Targeting HIF-1 for cancer therapy. Nat Rev Cancer 2003;3:721-32. http://www.ncbi.nlm.nih.gov/pubmed/13130303.

22. EPSTEIN AC, GLEADLE JM, MCNEILL LA, HEWITSON KS, O'ROURKE J, MOLE DR, et al. C. elegans EGL-9 and mammalian homologs define a family of dioxygenases that regulate HIF by prolyl hydroxylation. Cell 2001;107:43-54. http://www.ncbi.nlm.nih.gov/pubmed/11595184.

23. PUGH CW, RATCLIFFE PJ. Regulation of angiogenesis by hypoxia: role of the HIF system. Nat Med. 2003;9:677-84. http://www.ncbi.nlm.nih.gov/pubmed/12778166.

24. OPS/OMS Chile - Nuevo informe Salud en las Américas +2017 de la OPS: en Chile la esperanza de vida al nacer es de 80 años para los hombres y 85 años para las mujeres. https://www.paho. org/chi/index.php?option=com_content $\&$ view $=$ article $\&$ id=956:nuevo-informe-salud-en-las-americas-2017-de-la-opsen-chile-la-esperanza-de-vida-al-nacer-es-de-80-anos-paralos-hombres-y-85-anos-para-las-mujeres\&Itemid=1005.

25. TANAKA T, ECKARDT K-U. HIF Activation against CVD in CKD: novel treatment opportunities. Semin Nephrol. 2018;38:26776. http://www.ncbi.nlm.nih.gov/pubmed/29753402.

26. SEMENZA GL. Hypoxia-inducible factor 1 and cardiovascular disease. Annu Rev Physiol. 2014;76:39-56. http://www.ncbi. nlm.nih.gov/pubmed/23988176.

27. SEMENZA GL. Oxygen sensing, hypoxia-inducible factors, 
and disease pathophysiology. Annu Rev Pathol Mech Dis. 2014;9:47-71.

28. WANG H, KEISER JA. Vascular endothelial growth factor upregulates the expression of matrix metalloproteinases in vascular smooth muscle cells: Role of flt-1. Circ Res. 1998;83:832-40.

29. ZOU J, FEI Q, XIAO H, WANG H, LIU K, LIU M, et al. VEGF-A promotes angiogenesis after acute myocardial infarction through increasing ROS production and enhancing ER stress-mediated autophagy. J Cell Physiol. 2019;234:17690-703.

30. LEE S, PAUL W, RYAN E, REENA D, STUART J. Early expression of angiogenesis factor in acute myocardial ischemia and infarction. EKGs Nurse Pract Physician Assist. 2000;342:626-33.

31. REY S, LEE K, WANG CJ, GUPTA K, CHEN S, MCMILLAN A, et al. Synergistic effect of HIF-1alpha gene therapy and HIF-1-activated bone marrow-derived angiogenic cells in a mouse model of limb ischemia. Proc Natl Acad Sci USA. 2009;106:20399-404. http://www.ncbi.nlm.nih.gov/pubmed/19948968.

32. MURRY CE, JENNINGS RB, REIMER KA. Preconditioning with ischemia: A delay of lethal cell injury in ischemic myocardium. Circulation. 1986;74:1124-36.

33. HEUSCH G, GERSH BJ. The pathophysiology of acute myocardial infarction and strategies of protection beyond reperfusion: A continual challenge. Eur Heart J. 2017;38:774-84.

34. GHAEMIAN A, NOURAEI SM, ABDOLLAHIAN F, NAGHSHVAR F, GIUSSANI DA, NOURAEI SAR. Remote ischemic preconditioning Ghaemian, A., Nouraei, S. M., Abdollahian, F., Naghshvar, F., Giussani, D. A., \& Nouraei, S. A. R. (2012). Remote ischemic preconditioning in percutaneous coronary revascularization: A double-blind randomized controlled cli. Asian Cardiovasc Thorac Ann. 2012;20:548-54.

35. JUNG F, PALMER LA, ZHOU N, JOHNS RA. Hypoxic regulation of inducible nitric oxide synthase via hypoxia inducible factor-1 in cardiac myocytes. Circ Res. 2000;86:319-25. http:// www.ncbi.nlm.nih.gov/pubmed/10679484.

36. CAI Z, ZHONG H, BOSCH-MARCE M, FOX-TALBOT K, WANG L, WEI C, et al. Complete loss of ischaemic preconditioning-induced cardioprotection in mice with partial deficiency of HIF-1. Cardiovasc Res 2007;77:463-70. http://www. ncbi.nlm.nih.gov/pubmed/18006459.

37. SARKAR K, CAI Z, GUPTA R, PARAJULI N, FOX-TALBOT $\mathrm{K}$, DARSHAN MS, et al. Hypoxia-inducible factor 1 transcriptional activity in endothelial cells is required for acute phase cardioprotection induced by ischemic preconditioning. Proc Natl Acad Sci USA. 2012;109:10504-9. http://www.ncbi.nlm. nih.gov/pubmed/22699503.

38. MENENDEZ-MONTES I, ESCOBAR B, PALACIOS B, GÓMEZ MJ, IZQUIERDO-GARCIA JL, FLORES L, et al. Myocardial VHL-HIF signaling controls an embryonic metabolic switch essential for cardiac maturation. Dev Cell. 2016;39:724-39. https://linkinghub.elsevier.com/retrieve/pii/ S1534580716308243.

39. KRISHNAN J, SUTER M, WINDAK R, KREBS T, FELLEY A, MONTESSUIT C, et al. Activation of a HIF1alpha-PPARgamma axis underlies the integration of glycolytic and lipid anabolic pathways in pathologic cardiac hypertrophy. Cell Metab. 2009;9:512-24. https://linkinghub.elsevier.com/retrieve/ pii/S1550413109001399.

40. MIRTSCHINK P, KRISHNAN J, GRIMM F, SARRE A, HÖRL M, KAYIKCI M, et al. HIF-driven SF3B1 induces KHK-C to enforce fructolysis and heart disease. Nature. 2015;522:444-9. http://www.ncbi.nlm.nih.gov/pubmed/26083752.

41. GUI L, LIU B, LV G. Hypoxia induces autophagy in cardiomyocytes via a hypoxia-inducible factor 1-dependent mechanism. Exp Ther Med. 2016; 11: 2233-9.

42. THUERAUF DJ, MARCINKO M, GUDE N, RUBIO M, SUSSMAN MA, GLEMBOTSKI CC. Activation of the unfolded protein response in infarcted mouse heart and hypoxic cultured cardiac myocytes. Circ Res. 2006;99:275-82.

43. ZHANG C, SYED TW, LIU R, YU J. Role of endoplasmic reticulum stress, autophagy, and inflammation in cardiovascular disease. Front Cardiovasc Med. 2017;4:1-8.

44. BELAIDI E, THOMAS A, BOURDIER G, MOULIN S, LEMARIÉ E, LEVY P, et al. Endoplasmic reticulum stress as a novel inducer of hypoxia inducible factor-1 activity: Its role in the susceptibility to myocardial ischemia-reperfusion induced by chronic intermittent hypoxia. Int J Cardiol. 2016;210:45-53. http://dx.doi.org/10.1016/j.ijcard.2016.02.096.

45. JAIN K, SURYAKUMAR G, GANJU L, SINGH SB. Amelioration of ER stress by 4-phenylbutyric acid reduces chronic hypoxia induced cardiac damage and improves hypoxic tolerance through upregulation of HIF-1 $\alpha$. Vascul Pharmacol. 2016;83:36-46. http://dx.doi.org/10.1016/j.vph.2016.03.004.

46. HÖLSCHER M, SCHÄFER K, KRULL S, FARHAT K, HESSE A, SILTER M, et al. Unfavourable consequences of chronic cardiac HIF-1 $\alpha$ stabilization. Cardiovasc Res. 2012;94:7786. https://academic.oup.com/cardiovascres/article-lookup/ doi/10.1093/cvr/cvs014. 
47. KUMAR S, WANG G, LIU W, DING W, DONG M, ZHENG $\mathrm{N}$, et al. Hypoxia-induced mitogenic factor promotes cardiac hypertrophy via calcium-dependent and hypoxia-inducible factor-1 $\alpha$ mechanisms. Hypertens 1979;72:331-42. https:// www.ahajournals .org/doi/10.1161/HYPERTENSIONAHA.118.10845.

48. SHYU K-G, LU M-J, CHANG H, SUN H-Y, WANG B-W, KUAN P. Carvedilol modulates the expression of hypoxia-inducible factor- $1 \alpha$ and vascular endothelial growth factor in a rat model of volume-overload heart failure. J Card Fail. 2005;11:152-9. http://www.ncbi.nlm.nih.gov/pubmed/15732037.

49. SANO M, MINAMINO T, TOKO H, MIYAUCHI H, ORIMO M, QIN Y, et al. p53-induced inhibition of Hif-1 causes cardiac dysfunction during pressure overload. Nature. 2007;446:4448. http://www.ncbi.nlm.nih.gov/pubmed/17334357.

50. CHAN MC, HOLT-MARTYN JP, SCHOFIELD CJ, RATCLIFFE PJ. Pharmacological targeting of the HIF hydroxylases - A new field in medicine development. Mol Aspects Med. 2016;47-48:54-75. http://www.ncbi.nlm.nih.gov/pubmed/26791432.

51. KILIAN EG, SADONI S, VICOL C, KELLY R, HULST K VAN, SCHWAIGER $M$, et al. Myocardial transfection of hypoxia inducible factor- $1 \alpha$ via an adenoviral vector during coronary artery bypass grafting. Circ J. 2010;74:916-24. http:// joi.jlc.jst.go.jp/JST.JSTAGE/circj/CJ-09-0594?from=CrossRef.

52. SPRICK JD, MALLET RT, PRZYKLENK K, RICKARDS CA. Ischaemic and hypoxic conditioning: potential for protection of vital organs. Exp Physiol. 2019;104:278-94. http://doi. wiley.com/10.1113/EP087122.

53. CREAGER MA, OLIN JW, BELCH JJF, MONETA GL, HENRY TD, RAJAGOPALAN S, et al. Effect of hypoxia-inducible factor-1alpha gene therapy on walking performance in patients with intermittent claudication. Circulation. 2011;124:1765-73. https://www.ahajournals.org/doi/10.1161/CIRCULATIONAHA.110.009407.
54. ZIMNA A, KURPISZ M. Hypoxia-inducible factor-1 in physiological and pathophysiological angiogenesis: applications and therapies. Biomed Res Int 2015;2015:549412. http://www. hindawi.com/journals/bmri/2015/549412/.

55. BESARAB A, CHERNYAVSKAYA E, MOTYLEV I, SHUTOV E, KUMBAR LM, GUREVICH K, et al. Roxadustat (FG4592): correction of anemia in incident dialysis patients. J Am Soc Nephrol. 2016;27:1225-33. http://www.jasn.org/lookup/ doi/10.1681/ASN.2015030241.

56. BESARAB A, PROVENZANO R, HERTEL J, ZABANEH R, KLAUS SJ, LEE T, et al. Randomized placebo-controlled dose-ranging and pharmacodynamics study of roxadustat (FG-4592) to treat anemia in nondialysis-dependent chronic kidney disease (NDD-CKD) patients. Nephrol Dial Transplant. 2015;30:1665-73. https://academic.oup.com/ndt/article-lookup/doi/10.1093/ndt/gfv302.

57. HOLDSTOCK L, MEADOWCROFT AM, MAIER R, JOHNSON BM, JONES D, RASTOGI A, et al. Four-week studies of oral hypoxia-inducible factor-prolyl hydroxylase inhibitor gsk1278863 for treatment of anemia. J Am Soc Nephrol. 2016; 27: 1234-44. http://www.jasn.org/lookup/doi/10.1681/ ASN.2014111139.

58. CAO Z, SHEN R, ZHANG X, CHENG G, YAN Z. Effects of remote ischemic preconditioning on acute myocardial injury in patients undergoing valve replacement. Irish J Med Sci. 2017;186:889-93. http://link.springer.com/10.1007/s11845016-1521-8.

59. SLOTH AD, SCHMIDT MR, MUNK K, KHARBANDA RK, REDINGTON AN, SCHMIDT M, et al. Improved long-term clinical outcomes in patients with ST-elevation myocardial infarction undergoing remote ischaemic conditioning as an adjunct to primary percutaneous coronary intervention. Eur Heart J. 2014;35:168-75. https://academic.oup.com/eurheartj/ article/35/3/168/492732.

60. HAUSENLOY DJ, CANDILIO L, EVANS R, ARITI C, JENKINS DP, KOLVEKAR S, et al. Remote ischemic preconditioning and outcomes of cardiac surgery. N Engl J Med. 2015;373:140817. http://www.nejm.org/doi/10.1056/NEJMoa1413534 